\title{
Conductive Path Formation by Aggregated Oxygen Ions in Titanium Dioxide: First-Principles Analyses
}

\author{
Lei $\mathrm{Li}^{1{ }^{1},}$, Wenshi $\mathrm{Li}^{1}$, Aimin $\mathrm{Ji}^{2}$, Ziou Wang ${ }^{1}$, Canyan Zhu², Lijun Zhang ${ }^{2}$, \\ Jianfeng $\mathrm{Yang}^{1}$, and Ling-Feng Mao, ${ }^{2, *}$ \\ ${ }^{1}$ Institute of Intelligent Structure and System, School of Electronics \& Information Engineering, \\ Soochow University, Suzhou 215006, P.R. China \\ ${ }^{2}$ Institute of Intelligent Structure and System, Soochow University, Suzhou 215006, P.R. China \\ "lei_li56@163.com, lingfengmao@suda.edu.cn
}

Keywords: Conductive path, O interstitials, Aggregated O-ions

\begin{abstract}
A first-principle study on the conductive path formation induced by the aggregated $\mathrm{O}$ ions in the defected titanium dioxide $\left(\mathrm{Ti}_{12} \mathrm{O}_{29}\right)$ is reported. The result illustrates that the $\mathrm{O}$ interstitials induce the aggregation of the O-ions. Thus, the formation of the conductive path is attributed to the continuous $\mathrm{O}-\mathrm{O}$ bonds among these aggregated $\mathrm{O}$-ions. In the I-V and $\mathrm{R}-\mathrm{V}$ curves with the transmission coefficients, we find the resistance difference of the $\mathrm{Pt} / \mathrm{Ti}_{12} \mathrm{O}_{29} / \mathrm{Pt}$ device.
\end{abstract}

\section{Introduction}

As the urgent demand for the miniaturization of microelectronics, people need search alternatives to current flash memory for next generation high-density universal memories. Resistance-switching memory with the metal-insulator-metal structure (MIM) could satisfy the demand due to the superior characteristics [1]. Huge numbers of physical analysis have been performed on the unknown mechanism of resistive switching. It could be mainly classified into the electrochemical metallization memories (ECM) and the valence change memories (VCM) [2]. However, the basic information about the composition of the conductive filament buried under the metal electrodes in both ECM and VCM has not been thoroughly understood. Kwon et al. reported some regions blew off by the compressed excess oxygen gas in the $\mathrm{Pt} / \mathrm{TiO}_{2} / \mathrm{Pt}$ stack after the forming process [1]. They felt that these regions might correspond to the locations where the conductive filaments have formed. We propose that large numbers of oxygen ions maybe aggregated under the blown-off regions. So, can these aggregated O-ions form the partial conductive path near the anode? This article aims at the first-principles study on the conductive path formation induced by the aggregated oxygen ions in the defected $\mathrm{TiO}_{2}$.

\section{Method}

The structure model of perfect rutile $\mathrm{TiO}_{2}$ is presented as a periodically repeated primitive cell (lattice parameters $\mathrm{a}=\mathrm{b}=4.594 \AA, \mathrm{c}=2.959 \AA)$. We first construct $1 \times 1 \times 6$ supercells $(\mathrm{a}=\mathrm{b}=4.594$ $\AA, \mathrm{c}=17.754 \AA$ ) as the initial structure model. Then, five oxygen interstitials are added in the $1 \times 1 \times 6$ supercells, as shown in Fig. 1(a). The structures are relaxed at $0 \mathrm{MV} / \mathrm{cm}$ as shown in Fig. 1(b), at $1.3 \mathrm{MV} / \mathrm{cm}$ in Fig. 1(c), and $2.6 \mathrm{MV} / \mathrm{cm}$ in Fig. 1(d). In Fig. 1, the gray balls, the red ball and the green balls indicate Ti-ions, $\mathrm{O}$-ions and $\mathrm{O}$ interstitials, respectively. 


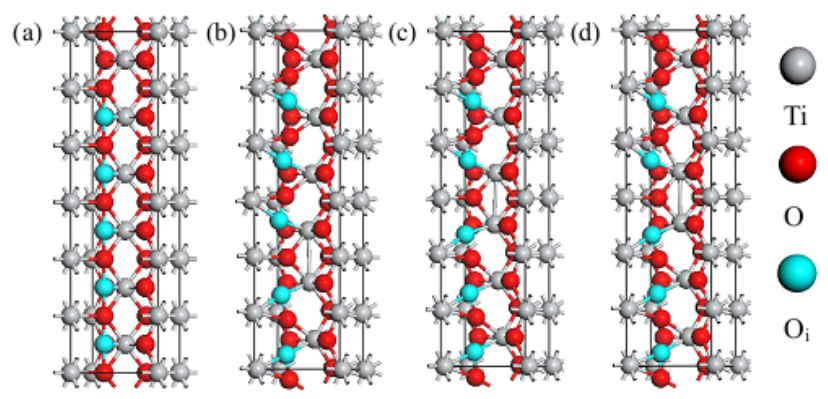

Fig. 1 Schematic diagram of the initial structure of the defected $\mathrm{TiO}_{2}$ with five $\mathrm{O}$ interstitials (a), the relaxed structure at $0 \mathrm{MV} / \mathrm{cm}$ (b), at $1.3 \mathrm{MV} / \mathrm{cm}$ (c), and $2.6 \mathrm{MV} / \mathrm{cm}(\mathrm{d})$.

The density functional theory calculations are performed on the $\mathrm{DMol}^{3}$ program with the spin polarized set [3-5]. The geometries are optimized using the double-numeric quality basis set (DNP) of 3.5, also with the PBE (Perdew-Burke-Ernzerhof) gradient-corrected function to describe the exchange and correlation effects [6]. Special sampling points in the Brillouin zone are used with the Monkhorst-Pack schemes of $3 \times 3 \times 2$ meshes [7]. A thermal smearing of $0.01 \mathrm{Ha}(1 \mathrm{Ha}=27.2114$ $\mathrm{eV}$ ) and a global orbital cutoff of $5.2 \AA$ are employed to accelerate the computational performance. The tolerances of energy, gradient, and displacement convergence for the geometric optimization and energy calculation were set to $1 \times 10^{-5} \mathrm{Ha}, 2 \times 10^{-3} \mathrm{Ha} / \AA$, and $5 \times 10^{-3} \AA$, respectively. We further use Virtual Nanolab program to calculate the transport coefficient with the DFT-PBE function at $300 \mathrm{~K}$. The cut-off of the grid mesh is set to 40 Hartree. The basis sets of double zetas and polarization orbitals (DZP) are performed in the transport simulations.

\section{Results and discussion}

Fig. 2 shows the partial density of states for the defected $\mathrm{TiO}_{2}$ with five $\mathrm{O}$ interstitials under 0 $\mathrm{MV} / \mathrm{cm}$ (a), $1.3 \mathrm{MV} / \mathrm{cm}$ (b), and 2.6 MV/cm (c). The curves with the light green color, the red color, and the blue color indicate the $\mathrm{p}$ states, the $\mathrm{d}$ states, and the sum states, respectively. In Fig. 2, the defect energy levels lie on the top of the valence band maximum (VBM), which shift upward the valence band edge to narrow the band gap to $0.625 \mathrm{eV}$. The defect energy levels mainly originate from the contribution of $\mathrm{O}-2 \mathrm{p}$ orbital in the O-O bonds. The Fermi energy locates at the energy of 0 $\mathrm{eV}$ above the valence band maximum. It means the p-type semiconductor characteristics. The peaks of the defect energy levels locate at $-0.5 \mathrm{eV}$ in Fig. 2(a), at $-1 \mathrm{eV}$ in Fig. 2(b), and at $-0.3 \mathrm{eV}$ in Fig. 2(c).
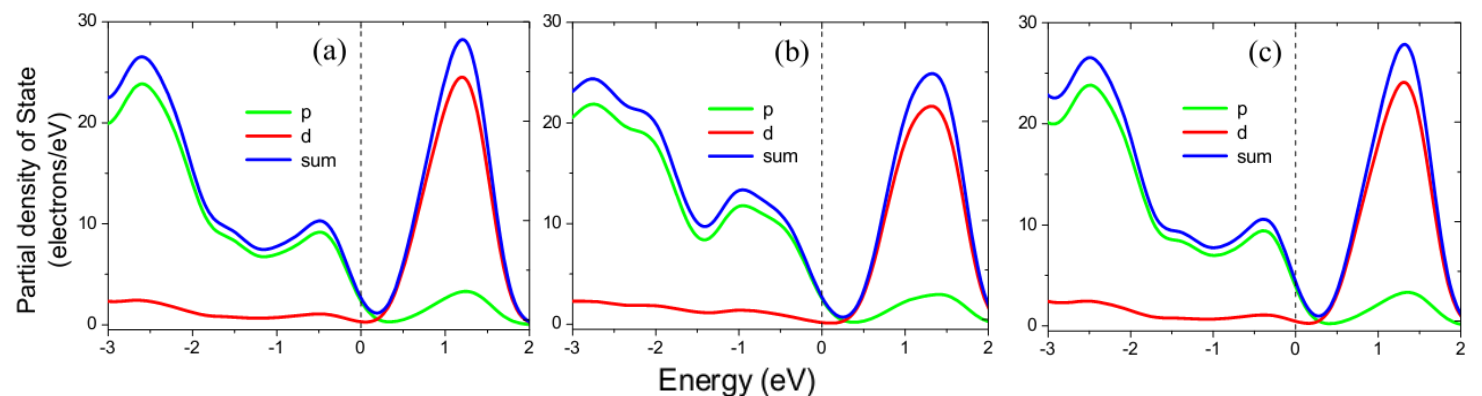

Fig. 2 Partial density of states for the defected TiO2 under $0 \mathrm{MV} / \mathrm{cm}$ (a), $1.3 \mathrm{MV} / \mathrm{cm}$ (b), and 2.6

$\mathrm{MV} / \mathrm{cm}(\mathrm{c})$.

Fig. 3 shows the deformation electron density in [110] for the defected $\mathrm{TiO}_{2}$ with five $\mathrm{O}$ interstitials under $0 \mathrm{MV} / \mathrm{cm}(\mathrm{a}), 1.3 \mathrm{MV} / \mathrm{cm}(\mathrm{b}), 2.6 \mathrm{MV} / \mathrm{cm}$ (c), and the corresponding white-black views (d-f).In Fig. 3 (a-c), the gray balls, the red ball and the green balls indicate Ti-ions, O-ions and $\mathrm{O}$ interstitials, respectively. We set the spectrum to the red-green-blue from -0.08 to 0.08 electron $/ \AA^{3}$; the losses of electrons are indicated as the red, while the electrons enrichment are indicated as the blue. It shows the formation of $\mathrm{O}-\mathrm{O}$ bonds because of the doping of the $\mathrm{O}$ interstitials. The Mulliken charges of the $\mathrm{O}$-ions in the $\mathrm{O}-\mathrm{O}$ bonds are in the range of $-0.336 \mathrm{eV} \sim$ $-0.414 \mathrm{eV}$ which are much fewer than them in other unbonding O-ions (in the range of $-0.781 \mathrm{eV} \sim$ $-0.961 \mathrm{eV})$. It means that the O-ions in the O-O bonds obtain fewer electrons than other O-ions do. 
At $0 \mathrm{MV} / \mathrm{cm}$ in Fig. 3(a), the $\mathrm{O}(7)$ interstitial is bonding to the lattice $\mathrm{O}(8)$ ion. It induces the formation of the Ti-Ti bond near the lattice $\mathrm{O}(6)$ ion. By contrast, as the impact of the electric field in Fig. 3(b-c), the $\mathrm{O}(7)$ interstitial form the new bond with the lattice $\mathrm{O}(6)$ ion. So, the Ti-Ti bond locates near the lattice $\mathrm{O}(8)$ ion. The O-O bonds delocalize the electron clouds which should localize at the O-ions in the Ti-O bonds. The electron deletion area with the red color are connected around the lattice $\mathrm{O}(6)$ ion or $\mathrm{O}(7)$ ion, which is also seen at the dark area connected by the white dash lines. It means the formation of the conduction path as the occurrence of the O-O bonds.

(a)

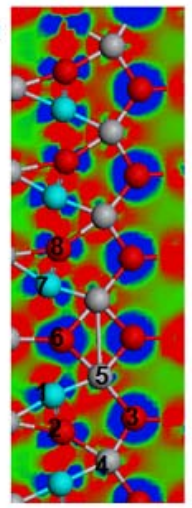

(b)

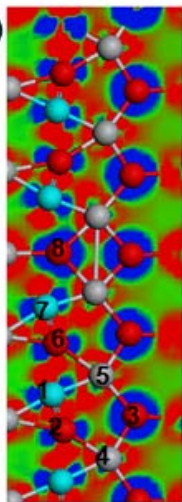

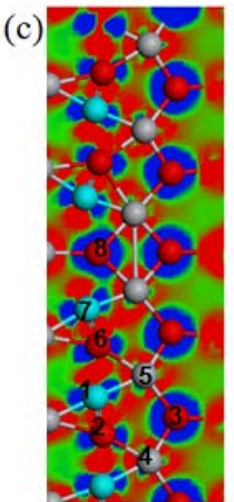
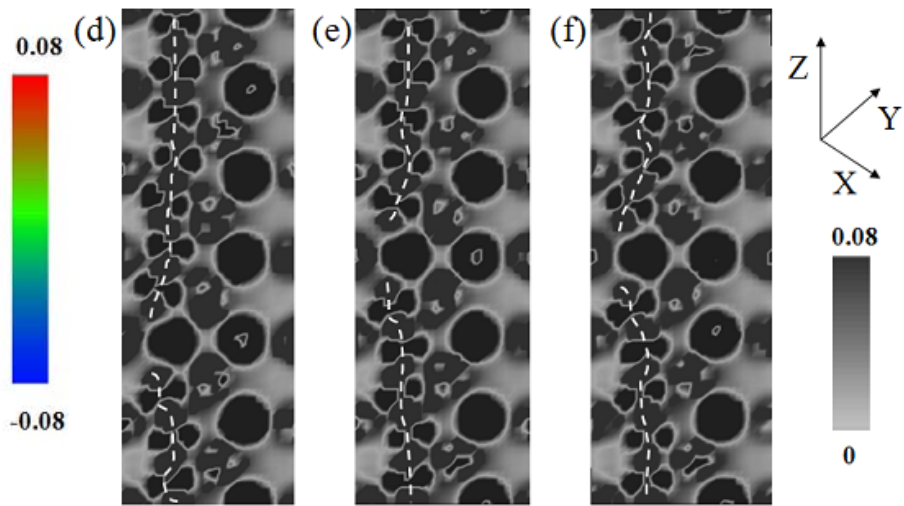

Fig. 3 Deformation electron density in [110] for the defected TiO2 with five O interstitials under 0 $\mathrm{MV} / \mathrm{cm}$ (a), $1.3 \mathrm{MV} / \mathrm{cm}$ (b), $2.6 \mathrm{MV} / \mathrm{cm}$ (c), and the corresponding white-black views (d-f).
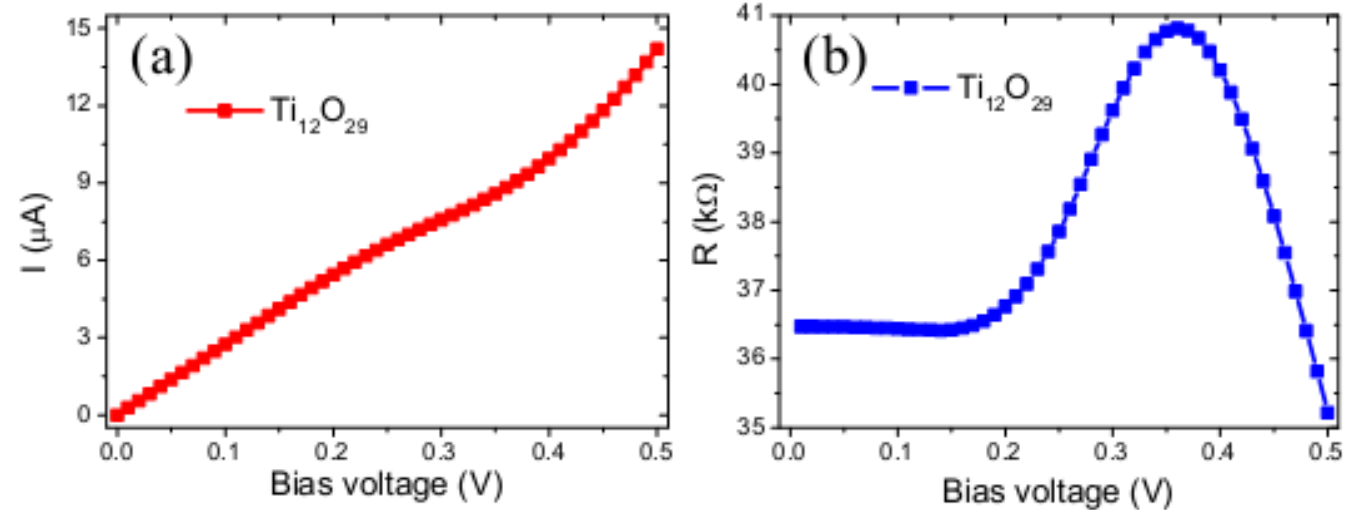

Fig. 4 I-V curves (a) and the R-V curves (b) of the Pt/Ti12O29/Pt device.

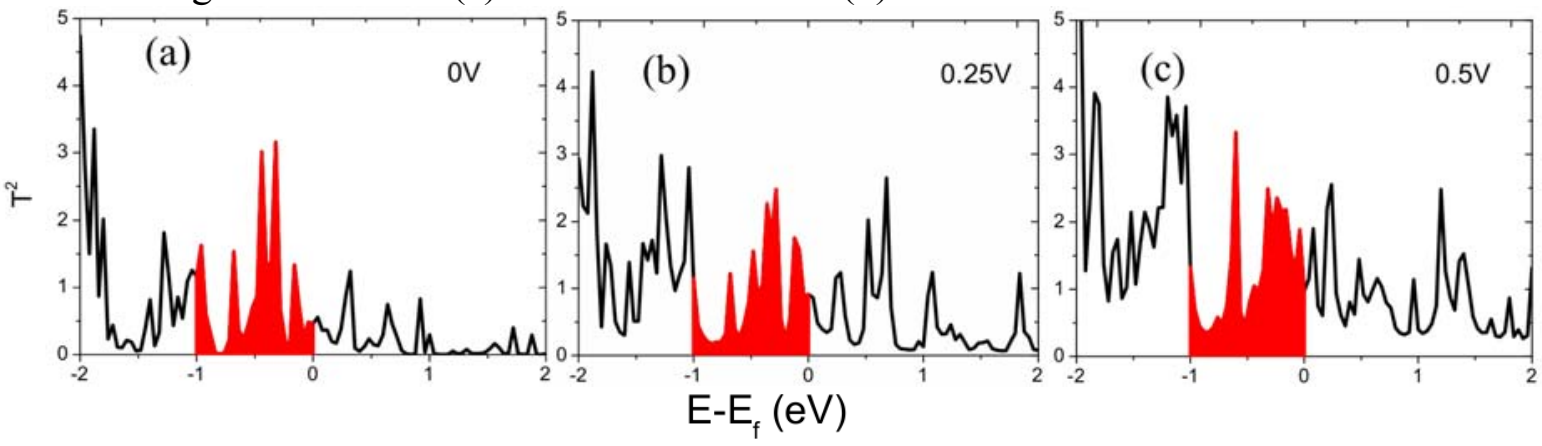

Fig 5 Transport coefficient of the Pt/Ti12O29/Pt device at $0 \mathrm{~V}(\mathrm{a}), 0.25 \mathrm{~V}$ (b), and $0.5 \mathrm{~V}$ (c).

Fig. 4 shows the $\mathrm{I}-\mathrm{V}$ curves and the $\mathrm{R}-\mathrm{V}$ curves of the $\mathrm{Pt} / \mathrm{Ti}_{12} \mathrm{O}_{29} / \mathrm{Pt}$ device. In Fig. 4(a), the current is also increased as the increasing of the bias voltage. By contrast, the resistance keeps steady at the initial stage of $0 \mathrm{~V}-0.2 \mathrm{~V}$, then increases at $0.2 \mathrm{~V}-0.36 \mathrm{~V}$, and decreases at $0.36 \mathrm{~V}-0.5 \mathrm{~V}$. The maximum resistance $(40.82 \mathrm{~K} \Omega)$ is at $0.36 \mathrm{~V}$, while the minimum $(35.22 \mathrm{~K} \Omega)$ at $0.5 \mathrm{~V}$.

Fig. 5 shows the transport coefficient of the $\mathrm{Pt} / \mathrm{Ti}_{12} \mathrm{O}_{29} / \mathrm{Pt}$ device at $0 \mathrm{~V}(\mathrm{a}), 0.25 \mathrm{~V}$ (b), and $0.5 \mathrm{~V}$ (c). In Fig. 2, we find that the defect energy levels locate at the range of $-1 \mathrm{eV}-0 \mathrm{eV}$. So, we could find the high transport coefficient at $-0.32 \mathrm{eV}$ in Fig. 5(a), $-0.28 \mathrm{eV}$ in Fig. 5(b), and $-0.6 \mathrm{eV}$ in Fig. $5(c)$, which are all induced by the defect energy levels of the $\mathrm{O}$ interstitials. So, the maximum of the transport coefficient at $-1 \mathrm{eV}-0 \mathrm{eV}$ is at $0.5 \mathrm{~V}$ in Fig. 5(c), the minimum is at $0.25 \mathrm{~V}$ in Fig. 5(b). 
Thus, the former leads to the lower resistance, while the latter is corresponding to the higher resistance.

\section{Conclusions}

We have reported the first-principles study on the conductive path induced by the aggregated $\mathrm{O}$-ions in defected $\mathrm{TiO}_{2}$. It shows that these $\mathrm{O}$-ions are aggregated in the relaxed structure as the $\mathrm{O}$ interstitial doping. It further leads to the formation of conductive path. The carriers in the conduction path are mainly composed of the holes. Moreover, the high transport coefficient determined by the defect energy levels as the doping of the $\mathrm{O}$ interstitials under the higher electric filed lead to the lower resistance at the higher bias voltage. This work can help to clarify the atom-level mechanism of conduction path in resistance random access memory [8].

\section{Acknowledgments}

The authors acknowledge the support from the National Natural Science Foundation of China under Grant Nos. 61076102 and 61272105, Natural Science Foundation of Jiangsu Province of China under Grant Nos. BK2012614 and BK20141196.

\section{References}

[1] D.H. Kwon, K.M. Kim, J.H. Jang, J.M. Jeon, M.H. Lee, G.H. Kim, X.S. Li, G.S. Park, B. Lee, S. Han, M. Kim, C.S. Hwang, Atomic structure of conducting nanofilaments in $\mathrm{TiO}_{2}$ resistive switching memory, Nat. Nanotechnol. 5 (2010) 148-153

[2] R. Waser, R. Dittmann, G. Staikov, K. Szot, Redox-based resistive switching memories-nanoionic mechanisms, prospects, and challenges, Adv. Mater. 21 (2009) 2632-2663

[3] M.D. Rasmussen, L.M. Molina, B. Hammer, Adsorption of $\mathrm{O}_{2}$ and oxidation of $\mathrm{CO}$ at $\mathrm{Au}$ nanoparticles supported by $\mathrm{TiO}_{2}$ (110), J. Chem. Phys. 120 (2004) 7673-7680

[4] B. Delley, From molecules to solids with the DMol3 approach, J. Chem. Phys. 113 (2000) 7756-7764

[5] M.Janousch, G.I. Meijer, U. Staub, B. Delley, S.F. Karg, B.P. Andreasson, Role of Oxygen Vacancies in Cr-Doped $\mathrm{SrTiO}_{3}$ for Resistance-Change Memory, Adv. Mater. 19 (2007) 2232-2235

[6] J.P. Perdew, K. Burke, M. Ernzerhof, Generalized gradient approximation made simple, Phys. Rev. Lett. 77 (1996) 3865

[7] H.J. Monkhorst, J.D. Pack, Special points for Brillouin-zone integrations, Phys. Rev. B 13 (1976) 5188

[8] K.M. Kim, D. S. Jeong, C. S. Hwang, Nanofilamentary resistive switching in binary oxide system: a review on the present status and outlook, Nanotechnology 22 (2011) 254002 